\title{
Sensitive Hemoglobin Concentration Sensor Based on Graphene-Plasmonic Nano-structures
}

\author{
Atefeh Chahkoutahi ${ }^{1} \cdot$ Farzin Emami $^{1}$ (1) $\cdot$ Esmat Rafiee $^{2}$
}

Received: 1 June 2021 / Accepted: 27 August 2021 / Published online: 21 September 2021

(c) The Author(s), under exclusive licence to Springer Science+Business Media, LLC, part of Springer Nature 2021

\begin{abstract}
In this paper, half-cylindrical-shaped rods are arranged in a row in order to form hemoglobin concentration sensors. The proposed structures can effectively detect hemoglobin concentrations in blood samples. Five individual structures based on graphene-plasmonic combinations are proposed and investigated. The proposed structures are made of different layers of Au, $\mathrm{Ag}, \mathrm{SiO}_{2}$, and graphene. Different plates and cylindrical-shaped graphene layers are introduced in the structures to improve the functionality (absorption peak value and wavelength) of the absorber (sensor). Adding more Au layers strengthens the confinement of the incident electromagnetic waves and improves the absorption factor. Also, in the proposed structures, for improving the results, the effects of the chemical potential of graphene layers and " $G$ " (graphene layer thicknesses) on the absorption peak and wavelength are considered. The final suggested structure indicates unity absorption peak and thus can be utilized in wide ranges of applications. As a refractive index bio-sensor, the structure is considered for detecting hemoglobin concentrations in blood samples which indicates a reasonable sensitivity factor of $570 \mathrm{~nm} / \mathrm{RIU}$.
\end{abstract}

Keywords Bio-sensor $\cdot$ Graphene $\cdot$ Hemoglobin Concentration $\cdot$ Plasmonic $\cdot$ Refractive index sensor

\section{Introduction}

Nowadays, optical bio-sensors have attracted excessive attentions. Optical bio-sensors indicate much impressive functionalities compared to the traditional ones. They acquire the advantages of higher sensitivity, lower cost, smaller sizes, and higher sensitivity precisions [1]. These sensors can specifically be applied in healthcare systems, biotechnology labs, and other scientific regions [2]. Optical bio-sensors can be the result of the graphene-plasmonic combinations [3]. Plasmonic-graphene nano-structures can operate based on surface plasmon resonances (SPR) [4-6]. Grapheneplasmonic combinations contain interesting specifications

Farzin Emami

emami@sutech.ac.ir

Atefeh Chahkoutahi

at.chahkoutahi@gmail.com

Esmat Rafiee

e.rafiee@alzahra.ac.ir

1 Electronic Department of, Nano-Optoelectronic Research Center, Shiraz University of Technology, Shiraz, Iran

2 Department of Electrical Engineering, Faculty of Engineering, Alzahra University, Tehran, Iran including high optical confinement [3], very low losses, and electromagnetic (EM) tunability, which make them great candidates for sensors in $\mathrm{THz}$ spectral range [7]. Plasmonic nano-structures are made of the combinations of metals and dielectrics, while graphene contains layers of carbon atoms being arranged in two-dimensional lattices [7, 8]. Surface plasmon resonance (SPR), which is the interaction between free electrons of a metal and the EM light wave, can conquer the limitations of light caused by the diffraction of light in nano-scaled dimensions (lower than the propagating wavelengths). As a result, structures made of graphene-plasmonic can effectively be used as absorbers $[9,10]$, refractive index and temperature sensors [11], or bio-sensors for detection of important elements like hemoglobin [8] and glucose [12]. Graphene-plasmonic-based nano-structures can be fabricated based on different techniques [13, 14]. The plasmonicgraphene-based sensors can be designed based on different approaches. Refractive index (RI)-based structures are good candidates for designing accurate and sensitive biosensors $[15,16]$. Interesting researches were reported considering different configurations of plasmonic-graphene nanostructures. As an example, optical properties of plasmonic materials in the shape of gold nano-rods were investigated by considering the finite element method (FEM) [17]. The spatially 
oscillatory patterns were observed on the surface as a result of plasmon-mode wave functions [17]. In another research [18], numerical investigations of tunable SPRs and diploe cavity plasmon modes of the scattering cross-section (SCS) on different configurations of nano-rods were considered by utilizing the FEM method. Considering the different properties of these structures, the solid-gold/gold-shell nano-rods can be tuned and controlled for producing specific wavelengths [18]. In another work, a sensor based on the plasmonic-dielectric combinations with different dielectric core plasmonic metamaterial was proposed and investigated [19]. The proposed structure functions based on the combination of lattice resonance, localized SPR, and cavity plasmon resonance modes and indicated very interesting and functional results [19].

In another research, an SPR biosensor for detection of blood glucose concentrations in the range of $25-175 \mathrm{mg} / \mathrm{dl}$ with RI range of 1 to 1.0007 was proposed [20]. The proposed sensor exhibited an appropriate sensitivity amount of $275.15^{\circ} /$ RIU. In recent researches, considering the COVID19 pandemic, graphene-based biosensors were suggested for virus detection as they were more economic, accurate, and fast [20]. In another study, a graphene-plasmonic biosensor was suggested and analyzed. The structure was based on the combination of silver nanoparticles and graphene film layers. Considering the graphene thickness of $9 \mathrm{~nm}$ in the structure, the $304.6 \%$ gain in sensitivity compared to nongraphene structure was obtained [21]. Also, plasmonic nanostructures with different shapes (of sphere, cubic, cylindrical) were proposed for hemoglobin concentration sensing [22]. The results indicated different resonance wavelengths for the three nano-particle shapes. It is declared that, by managing and engineering tunable properties of graphenebased nano-structures, they can be effectively used as biosensors for different applications [23]. In another research, graphene-gold combination structure is proposed for detecting bio-molecules and biological cells like blood, glucose, and other elements, considering their refractive indices [24].
Table 1 Geometric parameters of Fig. 1

\begin{tabular}{ll}
\hline Parameter & Value $(\mathrm{nm})$ \\
\hline$R$ & 650 \\
$t$ & 20 \\
$d$ & 1400 \\
$g$ & 0.34 \\
$p$ & 750 \\
\hline
\end{tabular}

In all of the mentioned reports, the applications of graphene-plasmonic nano-structures in sensors and especially biosensors were stated and considered. In this work, novel sensitive biosensors based on graphene-plasmonic combinations are proposed.

\section{Geometry, Theoretical Model, and Numerical Method}

The first proposed structure is a combination of graphene, $\mathrm{Au}, \mathrm{Ag}$, and $\mathrm{SiO}_{2}$ layers as depicted in Fig. 1 .

The structure is consisted of the periodic pattern of halfcylindrical-shaped arrays of Au rods placed above Ag, graphene, and $\mathrm{SiO}_{2}$ layers. The geometric parameters of Fig. 1 are tabulated in Table 1.

Numerical simulations were conducted by using twodimensional finite element-based (FE) software (COMSOL Multiphysics 5.5). As the length of the half-cylindrical nanorods is much longer than their diameters, the 3-D model can be replaced by a 2-D model (with approximately the same results) $[25,26]$. These simplifications were done for reducing the time and decreasing the computer resources needed for simulations [25, 26].

In the structure as shown in Fig. 1a, a plane wave polarized in $z$-axis with $1-\mathrm{W}$ power is applied as the incident field. The periodic boundary condition is considered in $x$-direction.

Considering the finite element (FEM) method, the field distributions at resonance wavelength of $1053 \mathrm{~nm}$, in Fig. 2a; non-resonance wavelength of $1040 \mathrm{~nm}$, in Fig. 2b; and the
Fig. 1 a Schematic of the first proposed structure. $\mathbf{b}$ View of the single array of the structure

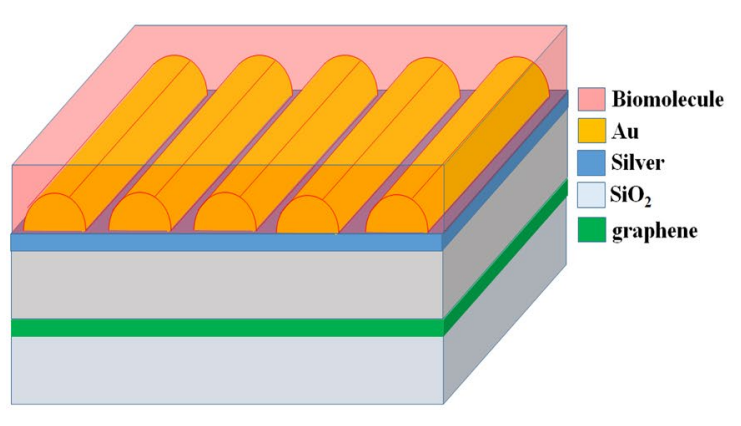

(a)

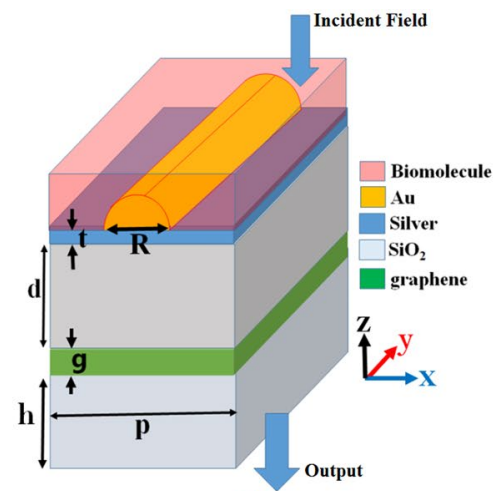

(b) 
Fig. 2 a Field distribution at $\lambda=1053 \mathrm{~nm}$. b Field distribution at $\lambda=1040 \mathrm{~nm}$. c Schematic of the absorption and reflection spectrum
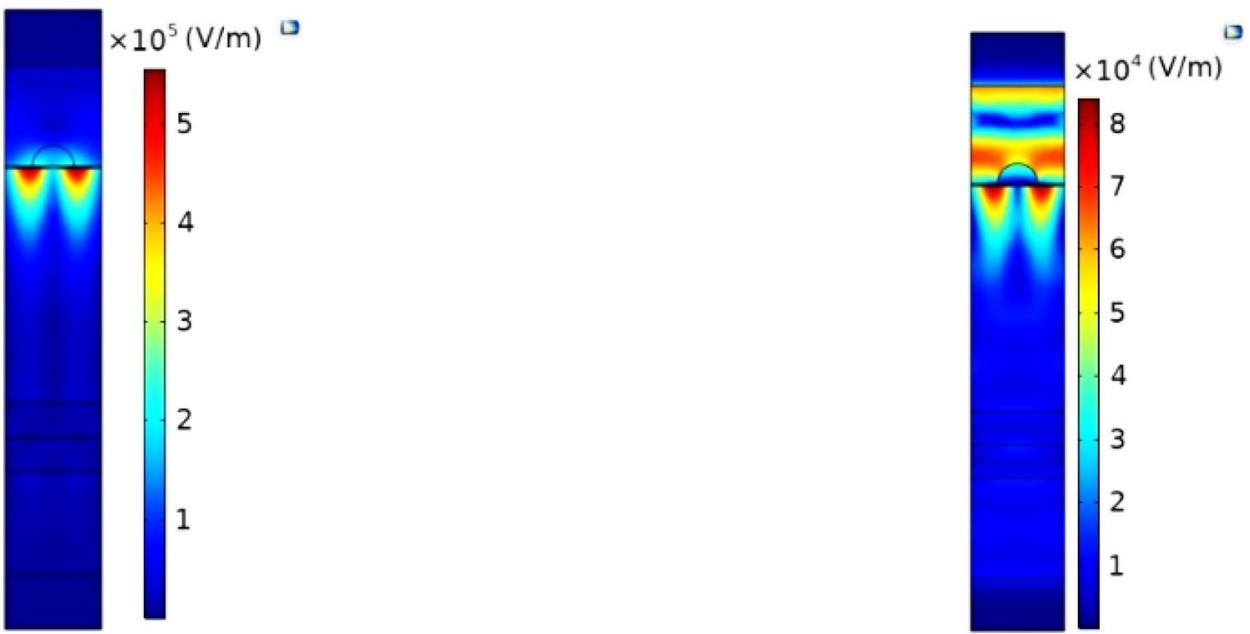

(a) (b)

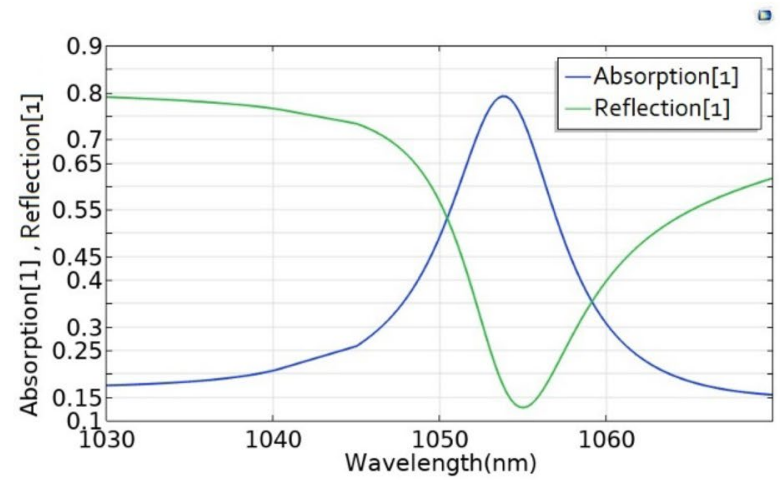

(c)

absorption and reflection spectrum, in Fig. 2c, are simulated and shown.

A simple equation can show the relation between the transmission, absorption, and reflection fields [16, 27]:

$A(\omega)=1-R(\omega)-T(\omega)$

Considering $T(\omega)=0$, then from (1) it is clear that $A(\omega)=1-R(\omega)$.

As it is considered to achieve the perfect absorption parameter (to have the proper sensor), the reflection parameter $R(\omega)$ should equal zero which leads to unity value for absorption $(A(\omega))$. As a result, the incident light wave would be thoroughly absorbed leading to the increment of the absorption peak value.

The surface conductivity of a graphene layer can be considered by the Kubo formula [16, 28]:

$\sigma_{g}\left(\omega, \mu_{c}, \Gamma, T\right)=\sigma_{g-\text { real }}+j \sigma_{g-\text { imag }}=\sigma_{\text {int } r a l}+\sigma_{\text {int } e r}$

where $\omega, \mu_{c}, \Gamma$, and $T$ indicate the operation frequency, the chemical potential, the phenomenological scattering rate, and the absolute temperature, respectively.
The intra-band and inter-band electro-photon scattering parameters can also be described as [16, 28]:

$\sigma_{\text {int } r a}=-j \frac{e^{2} k_{B} T}{\pi \hbar^{2}(\omega-j 2 \Gamma)} \times\left[\frac{\mu_{c}}{k_{B} T}+2 \ln \left(\exp \left(-\frac{\mu_{c}}{k_{B} T}\right)+1\right)\right]$

$\sigma_{\text {int } e r}=-j \frac{e^{2}}{4 \pi \hbar} \ln \left[\frac{2\left|\mu_{c}\right|-(\omega-j 2 \Gamma) \hbar}{2\left|\mu_{c}\right|+(\omega-j 2 \Gamma) \hbar}\right]$

where $k_{B}, T$, and $e$ are Boltzmann constant, temperature, and electron charge, respectively.

Drude model can introduce the dielectric function of the metals as [29-31]:

$\varepsilon(\omega)=\varepsilon_{\infty}-\frac{\omega_{p}^{2}}{\omega^{2}-j \omega \gamma}$

where $\varepsilon_{\infty}=3.7$ (dielectric constant at infinite frequency), $\gamma=0.018 \mathrm{eV}$ (collision plasma angular frequency), and $\omega_{p}=9.1 \mathrm{eV}$ (bulk plasma angular frequency) [29-31].

As can be seen from Fig. 2 (c), the absorption peak of 0.8 at $1053 \mathrm{~nm}$ is obtained. To improve the absorption peak 
Fig. 3 a Schematic of the second proposed structure. $\mathbf{b}$ View of the single array of the structure

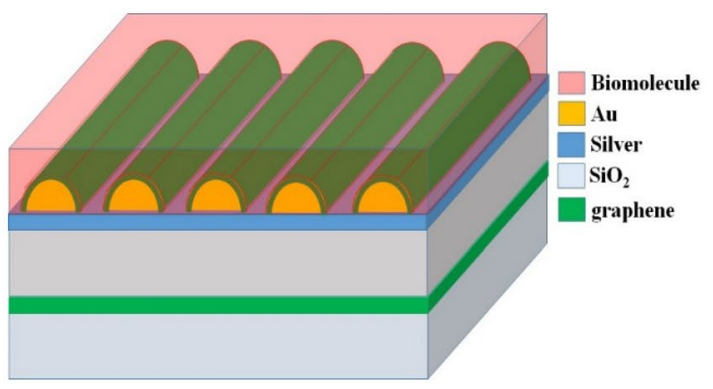

(a)

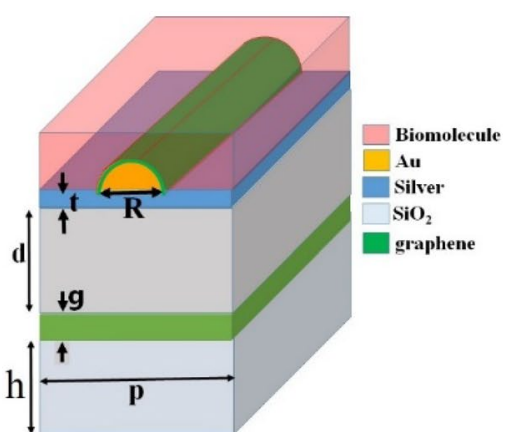

(b) and functionality of the structure, other combinations of graphene and plasmonic layers would be presented.

\section{Results and Discussion}

In this section, 4 different structures based on the combinations of graphene-plasmonic layers in the shape of halfcylindrical arrays are presented. It is important to notice that considering more layers of graphene and Au can affect the characteristics of the structures and increase their absorption peaks $[27,32]$. Therefore, their effects on the absorption spectrum will be discussed. The first structure was considered and evaluated in Fig. 1. The absorption peak value of 0.8 at wavelength of $1053 \mathrm{~nm}$ was achieved. The following proposed structures are proposed in order to improve the functionality of the structure by enhancing the absorption peak.

\section{Second Structure}

The second proposed structure is shown in Fig. 3. In this structure, a graphene layer is considered above the halfcylindrical arrays.
In this structure, another layer of graphene is coated above the Au half-cylindrical-shaped layers. The height of "h" equals $495 \mathrm{~nm}$. The thickness of the added graphene layer is the denoted by "g" and equals to $0.34 \mathrm{~nm}$.

By considering different values for the chemical potential from 0.3 to $1.5 \mathrm{eV}$, the absorption spectrum would be achieved as shown in Fig. 4.

Comparing Fig. 4 with Fig. 2c, the absorption peak value is increased from 0.8 to 0.88 . Increment of the absorption peak by adding a graphene layer can be the result of lowering the losses and enhancing the confinement of light. In fact, adding more graphene and plasmonic layers can increase the confinement of light and decrease the losses, which leads to higher peak values; this phenomenon continues until $N$ becomes more than 3 , where $N$ indicates the number of graphene layers. For $N>3$, the absorption peak would be decreased. In this condition, the graphene loss surpasses the metal loss; therefore, more energy can pass to outer layers and causes the absorption peak to descend [3, 32]. As a result, maximum 3 graphene layers are considered in the following structures.

As can be seen from Fig. 4, by increasing $\mu_{c}$, the absorption peak wavelength is shifted to the lower values. This result can be described by the circuit theory. In the circuit
Fig. 4 Schematic of the absorption spectrum versus wavelength for different values of $\mu_{c}$

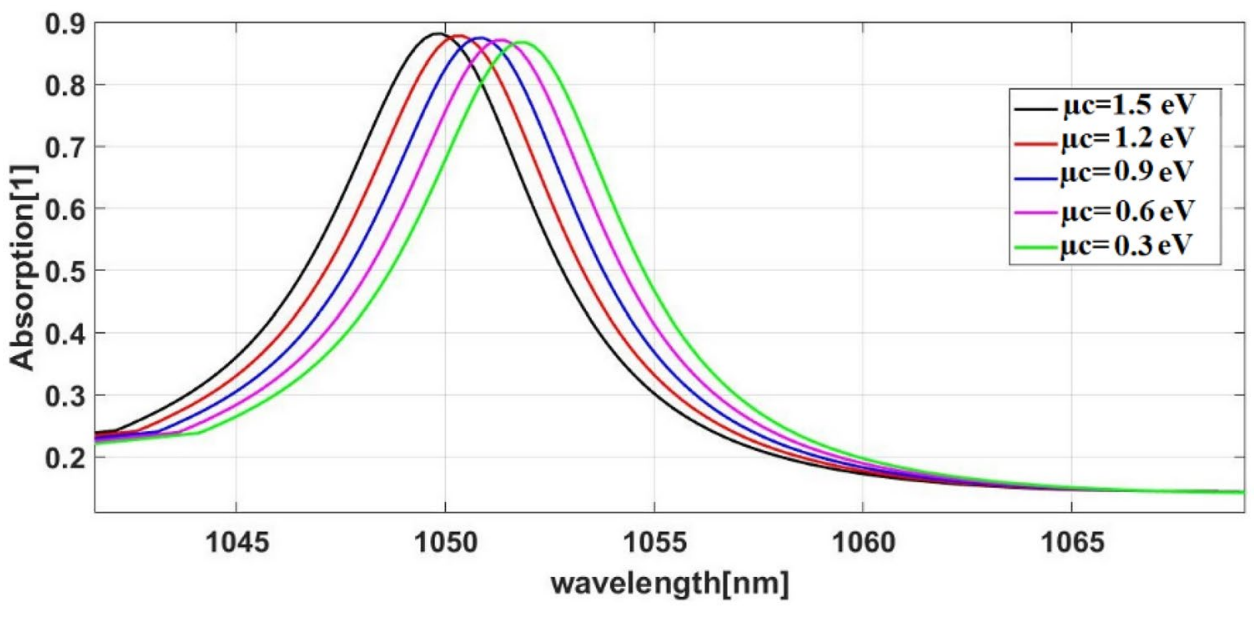




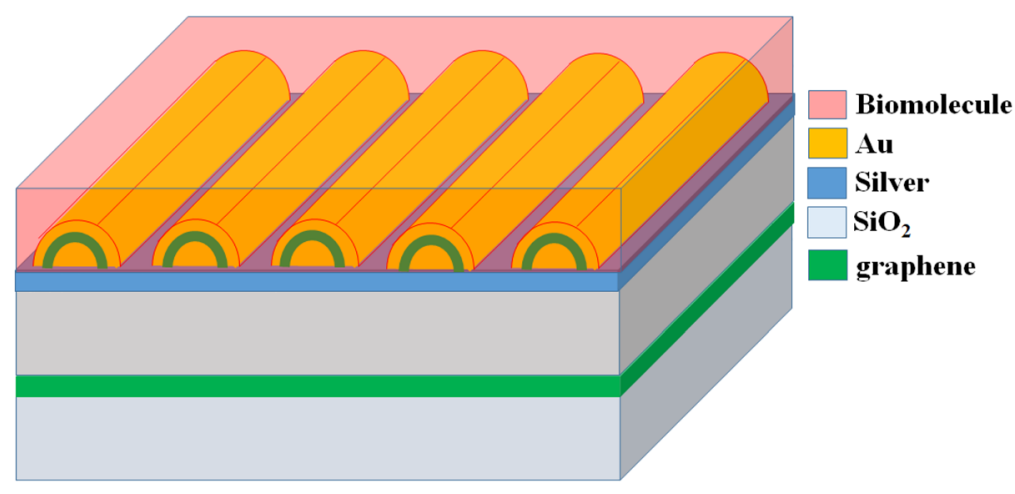

(a)

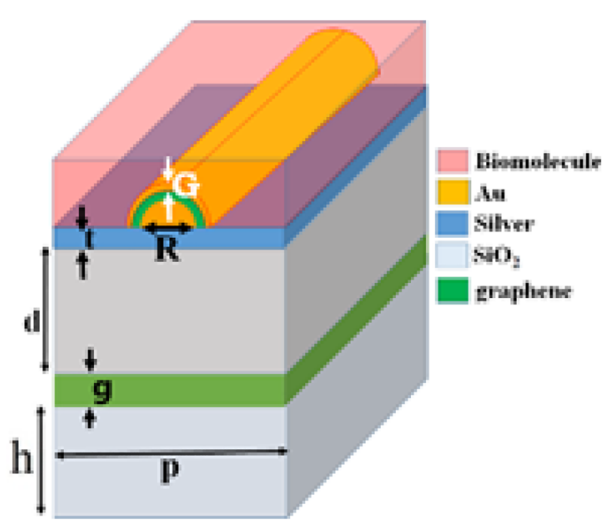

(b)

Fig. 5 a Schematic of the third proposed structure. b View of the single array of the structure

theory, graphene is characterized as a shunt admittance which can be varied by the geometrical parameters $g, p$, and chemical potential $\mu_{c}[16,33]$. Thus, altering $\mu_{c}$ can change the absorption peak wavelength according to [16, 33, 34]:

$\lambda=2 \pi c \sqrt{L C}$

where $c, L$, and $C$ are the speed of the light in vacuum, capacitance, and inductance of the circuit respectively. As can be concluded, increasing $\mu_{c}$ would decrease $L$ [35], which would decrease the absorption peak wavelength. By varying $\mu_{c}$ from 0.3 to $1.5 \mathrm{eV}$, the wavelength would be shifted from 1053 to $10,495 \mathrm{~nm}$. Therefore, by selecting the appropriate $\mu_{c}$, different resonance wavelengths for different applications can be achieved.

In this structure, by adding another layer of graphene, the absorption peak is improved. This happens due to the decrease of the Au loss and excessive confinement of the light in the Au layer.

\section{Third Structure}

The third proposed structure is shown in Fig. 5.

In this structure, a layer of $\mathrm{Au}$ is coated above the graphene layer with the thickness of "G." As discussed in the previous sections, adding an Au layer leads to the increased confinement of light which increases the absorption peak $[3,32]$. To discuss the effects of the added Au layer, its effects on the absorption spectrum are studied. The simulation results are plotted in Fig. 6.

As can be seen, by increasing "G," the absorption peak wavelength is shifted to the higher values. This happens due to the fact that increasing " $G$ " would increase the
Fig. 6 Schematic of the absorption spectrum versus wavelength for different values of " $\mathrm{G}$ "

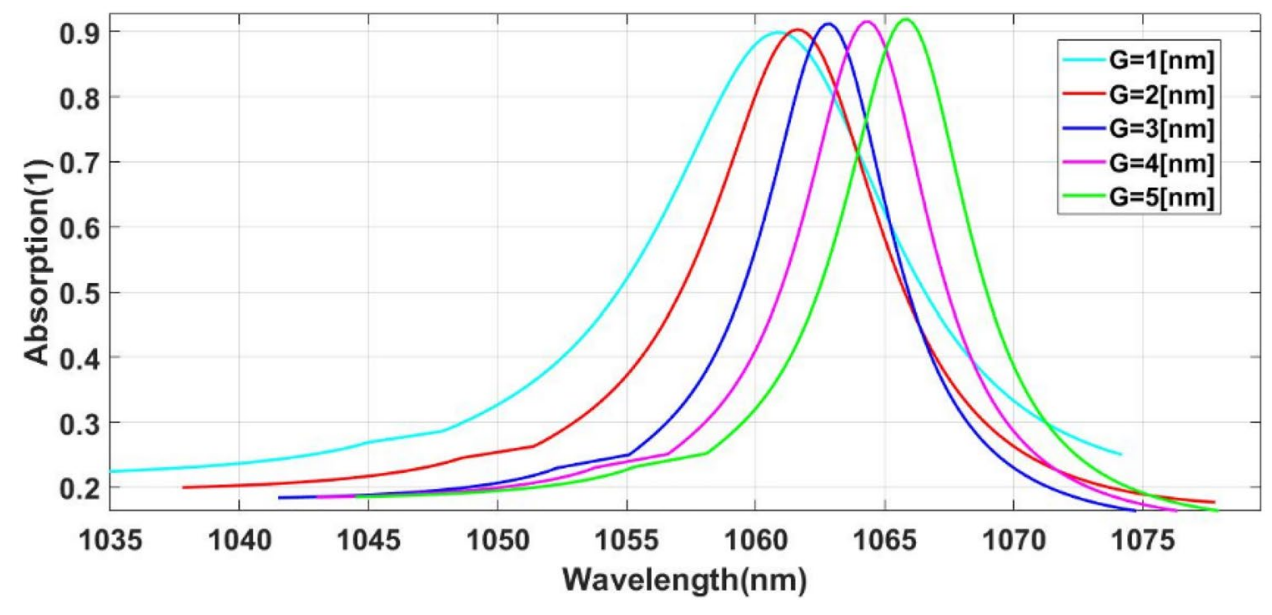


Fig. 7 a Schematic of the fourth proposed structure. $\mathbf{b}$ View of the single array of the structure

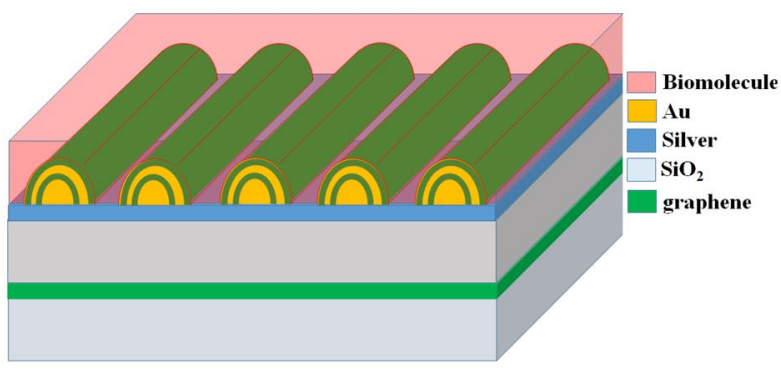

(a)

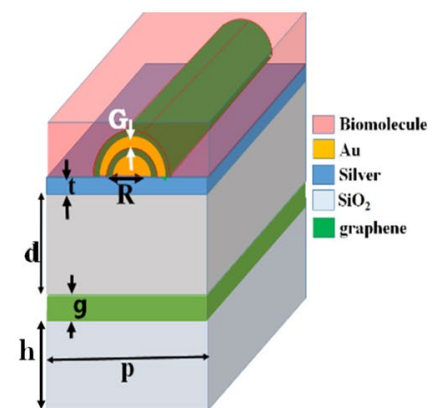

(b) capacitance; thus, according to (6), the wavelength would be shifted to the higher values $[16,33]$.

\section{Fourth Structure}

The fourth proposed structure is shown in Fig. 7.

In this structure, another layer of graphene is covered above the Au layer with the thickness of "g" (all the graphene layers' thickness equal "g"). For this structure, by changing the chemical potential, the absorption spectrum can be depicted in Fig. 8.

As can be seen, increasing $\mu_{c}$ would increase the absorption peak value and decrease the absorption peak wavelength. It was stated in the previous sections that increasing $\mu_{c}$ would decrease the inductance amount $(L)$ as the shunt admittance of the circuit theory $[16,34]$. Therefore, the absorption peak value would be about 0.98 for $\mu_{c}=1.5 \mathrm{eV}$ at $\lambda=1057.5 \mathrm{~nm}$.

\section{Fifth Structure}

The last proposed structure is shown in Fig. 9.

In this structure, another layer of graphene is positioned under the Au-graphene half-cylindrical-shaped arrays with the thickness of "g." The effects of " $\mu_{c}$ " on the absorption spectrum for the last proposed structure are plotted in Fig. 10.

As previously mentioned, " $\mu_{c}$ " affects the equivalent inductance " $L$ " of the circuit theory (increasing " $\mu_{c}$ " decreases " $L$ "), which leads to the blue shift of the absorption peak wavelength (shifts the wavelength to lower amounts). In the last structure, the absorption peak reaches unity. As a result, this structure can be considered the bio-sensor for detecting hemoglobin concentrations.

To consider the structure as the biosensor, the absorption spectrum for different concentrations of hemoglobin with various refractive indices is shown in Fig. 11.

These refractive indices of $1.335,1.34,1.36$, and 1.38 are related to different hemoglobin concentrations in blood samples $(n=1.335$ for $3.5 \mathrm{~g} / \mathrm{dl}, n=1.34$ for $10.6 \mathrm{~g} / \mathrm{dl}, n=1.36$ for $16.5 \mathrm{~g} / \mathrm{dl}, n=1.385$ for $28.7 \mathrm{~g} / \mathrm{dl}[15,36])$.

As can be seen in Fig. 11, increasing RI would shift the absorption peak wavelength to higher amounts $[3$, $16,37]$. Considering the sensitivity relation $[\Delta \lambda / \Delta n$ $(\mathrm{nm} / \mathrm{RIU})]$, the sensitivity value of $570 \mathrm{~nm} / \mathrm{RIU}$ can be achieved for this sensor. Table 2 indicates the comparison between our proposed sensors with some previously published works.
Fig. 8 Schematic of the absorption spectrum versus wavelength for different values of " $\mu_{c}$ "

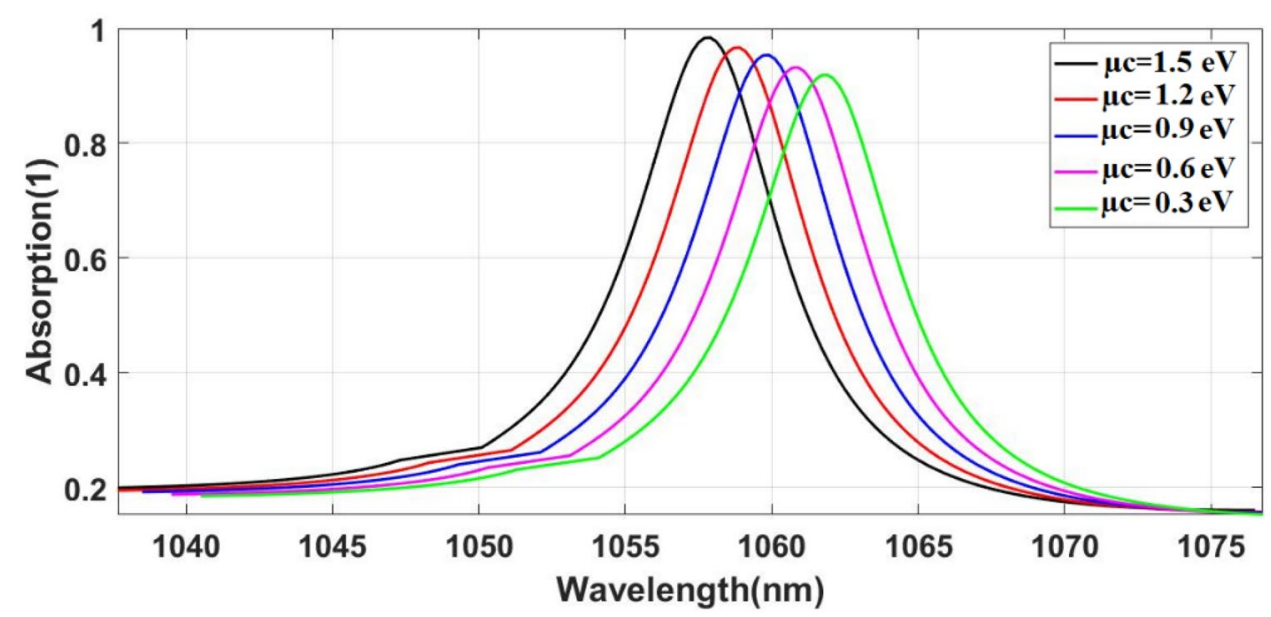


Fig. 9 a Schematic of the last proposed structure. $\mathbf{b}$ View of the single array of the structure

Fig. 10 Schematic of the absorption spectrum versus wavelength for different values of " $\mu c$ "

Fig. 11 Absorption spectrum versus wavelength for different hemoglobin concentrations

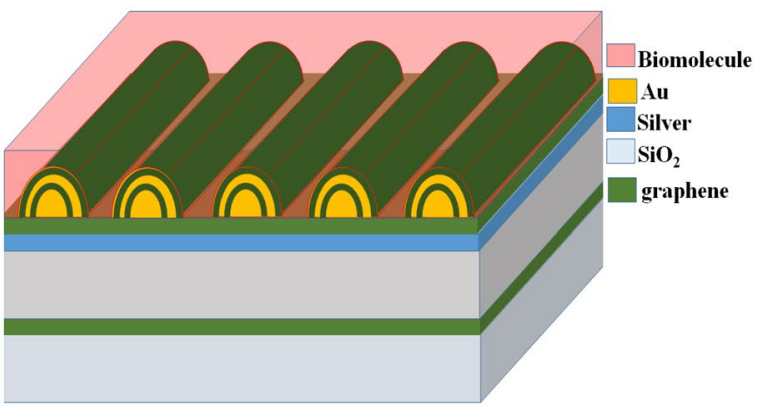

(a)

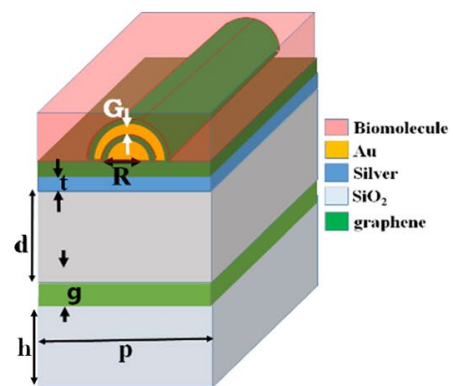

(b)
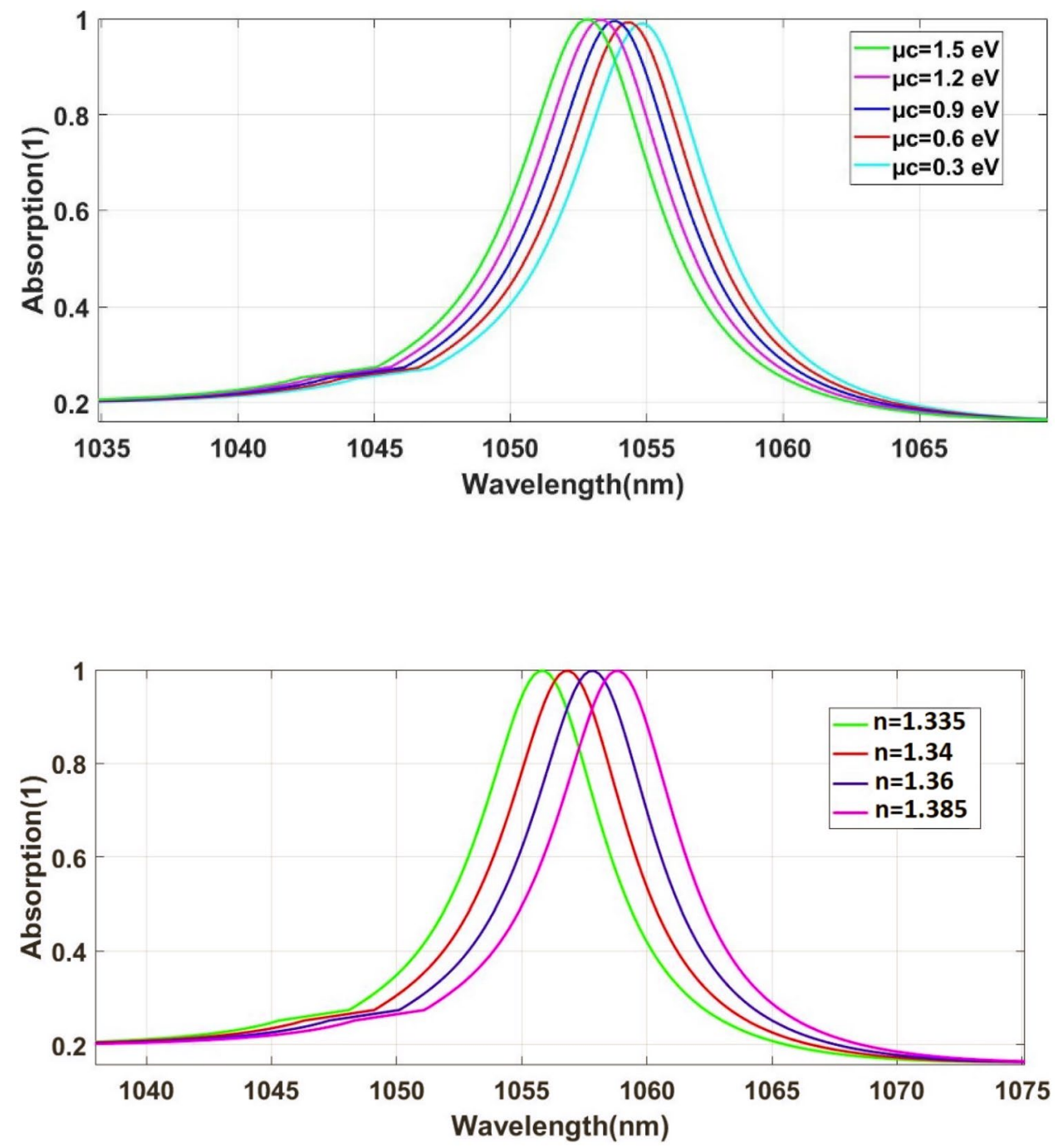
Table 2 Comparison of our proposed sensor with previous works

\begin{tabular}{ll}
\hline References & $\begin{array}{l}\text { Sensitivity } \\
\text { (nm/RIU) }\end{array}$ \\
\hline$[38]$ & 200 \\
{$[39]$} & 223.66 \\
{$[40]$} & 225 \\
{$[41]$} & 280 \\
{$[42]$} & 517.9 \\
Our proposed sensor & 570 \\
\hline
\end{tabular}

\section{Conclusion}

Five different configurations based on graphene-plasmonic nano-structures were considered for bio-sensing applications. These structures were made of half-cylindricalshaped rods of graphene and Au which were organized on different layers of $\mathrm{Ag}, \mathrm{SiO}_{2}$, and graphene. In order to improve the functionality of the structures, effects of chemical potential and graphene layer's thicknesses on the absorption peak and wavelength were also studied. By applying more cylindrical-shaped layers of Au and graphene, the absorption peaks experienced higher values. The final suggested structure, which showed unity absorption peak, was considered the refractive index sensor for detecting hemoglobin concentrations in blood samples. By considering RI values of $n=1.335$ for $3.5 \mathrm{~g} / \mathrm{dl}, n=1.34$ for $10.6 \mathrm{~g} / \mathrm{dl}, n=1.36$ for $16.5 \mathrm{~g} / \mathrm{dl}, n=1.385$ for $28.7 \mathrm{~g} / \mathrm{dl}$, the acceptable sensitivity factor of $570 \mathrm{~nm} / \mathrm{RIU}$ was achieved. As a result, the proposed structures can be interesting and great candidates for the detection of different bio-elements and tissues and can be appropriate bio-sensors.

Author Contribution Author contributions are equal.

\section{Declarations}

Ethics Approval Ethics approval is completely considered and applied.

Consent to Participate Consent to participate is checked.

Consent for Publication Consent for publication is checked.

Conflict of Interest The authors declare no competing interests.

\section{References}

1. El barghouti M et al (2020) Design of silver nanoparticles with graphene coatings layers used for LSPR biosensor applications. Vacuum 180:109497
2. Singh P (2017) Surface plasmon resonance: a boon for viral diagnostics, In book: Reference Module in Life Sciences

3. Hajati Y (2020) Tunable broadband multiresonance graphene terahertz sensor. Opt Mater 101:109725. https://doi.org/10.1016/j. optmat.2020.109725

4. Chau YF et al (2009) Surface plasmon effects excitation from three-pair arrays of silver-shell nanocylinders. Phys Plasmas 16:022303

5. Ho YZ et al (2012) Tunable plasmonic resonance arising from broken-symmetric silver nanobeads with dielectric cores. J Opt 14:114010

6. Chau YF (2009) Surface plasmon effects excited by the dielectric hole in a silver-shell nanospherical pair. Plasmonics 4:253-259

7. Emami F et al (2019) Plasmonic multi channel filter based on split ring resonators: application to photothermal therapy. Photonics Nanostructures: Fundam Appl 33:21-28

8. Sharma AK, Gupta J (2018) Graphene based chalcogenide fiberoptic evanescent wave sensor for detection of hemoglobin in human blood. Opt Fiber Technol 41:125-130

9. Chau YF et al (2020) Perfect dual-band absorber based on plasmonic effect with the cross-hair/nanorod combination. Nanomaterials 10:493

10. Chau YF et al (2018) Plasmonic effects in composite metal nanostructures for sensing applications. J Nanopart Res 20:190

11. Chau YF et al (2020) Enhancing plasmonic effect in periodic nanometal square prisms with fences and cavities for refractive index and temperature sensing applications. J Nanopart Res 22:297

12. Panda A et al (2020) Performance analysis of graphene-based surface plasmon resonance biosensor for blood glucose and gas detection. Appl Phys A 126. https://doi.org/10.1007/ s00339-020-3328-8

13. Wang $X$ et al (2014) Fabrication techniques of graphene nanostructures. Nanofabrication and its application in renewable energy pp 1-30. https://doi.org/10.1039/9781782623380-00001

14. Bonaccorso F et al (2012) Production and processing of graphene and 2d crystals. Mater Today 15:564-589

15. Rahman MS et al (2020) Enhanced performance of SnSegraphene hybrid photonic surface plasmon refractive sensor for biosensing applications. Photonics Nanostructures: Fundam Appl 39:100779. https://doi.org/10.1016/j.photonics.2020. 100779

16. Emami F et al (2021) Graphene-metal nanostructure as opto-fluid sensor. Optik Available online 10 March 166713. https://doi.org/ 10.1016/j.ijleo.2021.166713

17. Chau YF et al (2009) Three-dimensional analysis of surface plasmon resonance modes on a gold nanorod. Appl Opt 48:617-622

18. Chau YF et al (2016) Tailoring surface plasmon resonance and dipole cavity plasmon modes of scattering cross section spectra on the single solid-gold/gold-shell nanorod. J Appl Phys 120:093110

19. Chau YF et al (2018) Depolying tunable metal-shell/dielectric core nanorod arrays as the virtually perfect absorber in the nearinfrared regime. ACS Omega 3:7508-7516

20. Sengupta J et al (2021) Graphene-based field-effect transistor biosensors for the rapid detection and analysis of viruses: a perspective in view of COVID-19. Carbon Trends 2:100011. https://doi. org/10.1016/j.cartre.2020.100011

21. El barghouti M., et al (2020) Design of silver nanoparticles with graphene coatings layers used for LSPR biosensor applications. Vacuum 180:109497. https://doi.org/10.1016/j.vacuum.2020. 109497

22. Heidarzadeh H (2020) Analysis and simulation of a plasmonic biosensor for hemoglobin concentration detection using noble metal nano-particles resonances. Opt Commun 459:124940. https://doi.org/10.1016/j.optcom.2019.124940 
23. Vermisoglou E (2020) Human virus detection with graphenebased materials. Biosens Bioelectron 166:112436. https://doi. org/10.1016/j.bios.2020.112436

24. Sadeghi $Z$ et al (2020) Highly sensitive Mid-infrared SPR biosensor for a wide range of bio-molecules and biological cells based on graphene-gold grating. Physica E: Low-dimens Syst and Nanostruct 119:114005. https://doi.org/10.1016/j.physe.2020.114005

25. Chau YF et al (2019) Strong and tunable plasmonic field coupling and enhancement generating from the protruded metal nanorods and dielectric cores. Results Phys 13:102290

26. Chau YF et al (2020) Ultrawide bandgap and high sensitivity of a plasmonic metal-insulator-metal waveguide filter with cavity and baffles. Nanomater 10:2030

27. Li H et al (2019) Dual-band, polarization-insensitive metamaterial perfect absorber based on monolayer graphene in the midinfrared range. Results Phys 13:102313. https://doi.org/10.1016/j. rinp. 2019.102313

28. Hanson GW (2008) Dyadic green's functions and guided surface waves for a surface conductivity model of graphene. J Appl Phys 103. https://doi.org/10.1063/1.2891452

29. Emami $F$ et al (2018) Design and simulation of a novel nano plasmonic split ring resonator filter. J Electromagn Waves Appl 32:1925-1938

30. Emami F, Rafiee E, Negahdari R (2019) Design and analysis of the novel plasmonic split ring resonator power splitter appropriate for photonic integrated circuits. J Optoelectron Adv Mater 21:163-170

31. Emami F et al (2019) Realization of all-optical plasmonic MIM split square ring resonator switch. Opt Quantum Electron 51

32. Zare MS et al (2019) A strong controllable absorber using graphene metal Nanostructure. J Mod Opt 66:7-16
33. $\mathrm{Xu} \mathrm{Z}$ et al (2018) Design of a tunable ultra-broadband terahertz absorber based on multiple layers of graphene ribbons. Nanoscale Res Lett 13

34. Zhang J et al (2018) A dual-band tunable metamaterial near-unity absorber composed of periodic cross and disk graphene arrays. IEEE Photonics J 10

35. Liu C et al (2018) Symmetrical dual D-shape photonic crystal fibers for surface plasmon resonance sensing. Opt Express 26

36. Sharma AK et al (2018) Graphene based chalcogenide fiber-optic evanescent wave sensor for detection of hemoglobin in human blood. Opt Fiber Technol 41:125-130

37. Chen X et al (2018) Surface plasmon resonance sensor based on a novel D-shaped photonic crystal fiber for low refractive index detection. IEEE Photonics J 10

38. Vahed H et al (2019) Sensitivity enhancement of SPR optical biosensor based on Graphene-MoS2 structure with nanocomposite layer. Opt Mater 88:161-166

39. Dey B et al (2021) Numerical design of high-performance WS2/ metal/WS2/graphene heterostructure based surface plasmon resonance refractive index sensor. Results Phys 23:104021

40. Sahraeian $\mathrm{S}$ et al (2021) Tunable terahertz absorber based on graphene-metal nanostructure as opto-fluid Sensor. Optik 242:166713

41. Pal A et al (2021) A theoretical analysis on sensitivity improvement of an SPR refractive index sensor with graphene and barium titanate nanosheets. Optik 231:166378

42. Ye Y et al (2019) Highly sensitive and tunable terahertz biosensor based on optical Tamm states in graphene-based Bragg reflector. Results Phys 15:102779

Publisher's Note Springer Nature remains neutral with regard to jurisdictional claims in published maps and institutional affiliations. 